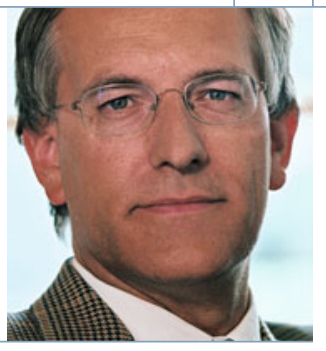

Martin Pöhlchen

\title{
Big Data - Chance für IT, Life Sciences und Healthcare?
}

DOI: $10.1007 /$ s 12268-014-0506-2

(C) Springer-Verlag 2014

Das weltweit erzeugte Datenvolumen wurde von der Computerfirma EMC für 2012 auf 2,8 Zettabyte $\left(10^{21}\right.$ Byte) geschätzt. In den Lebenswissenschaften befinden sich in den wichtigsten Genomdatenbanken bereits über 15 Petabyte ( $10^{15}$ Byte) an Daten, in der Proteomdatenbank ProteomicsDB über drei Terabyte; die chemische Datenbank ChemSpider zählt 26 Millionen unterschiedliche Moleküle aus über 400 Datenbanken auf. Täglich kommen zurzeit im US-Gesundheitssystem 15 Petabyte an Daten hinzu.

Schlagwort „Big Data“: Der Begriff wird durch ein ansteigendes Volumen (volume) der Daten, eine ansteigende Geschwindigkeit (velocity), mit der Daten erzeugt und verarbeitet werden, und eine steigende Vielfalt (variety) der erzeugten Daten charakterisiert [1]. Am Ende steht die Frage nach dem Wert (value) von Big Data - dem Wert für die Verbesserung der Patientenversorgung, für Prävention und Therapie sowie die personalisierte Medizin bei gleichzeitigen Kosteneinsparungen im Gesundheitswesen.

80 Prozent der Daten liegen als nicht strukturierte Daten vor: In den Lebenswissenschaften ist das z. B. ein Großteil der Hochdurchsatzscreening-Daten, präklinischen, klinischen Daten, sonstigen Forschungsdaten und Pharmakovigilanzdaten. Erschwert wird die Handhabung der Daten dadurch, dass die unterschiedlichen Datensätze aus verschiedenen Bereichen mit unterschiedlichen Eigentümern und Rechten stammen. Beim Scan eines Organs fallen in einer Sekunde zehn Gigabyte an Rohdaten an [2]. Die Geschwindigkeit, mit der Unmengen verschiedener Daten anfallen und in nahezu Echtzeit ausgewertet werden müssen, erfordert neue Datenbankarchitekturen, Algorithmen und Geschäftsprozesse.
Laut einer Analyse von McKinsey sind jedoch durch Big Data-Anwendungen Einsparungen im US-Gesundheitswesen von bis zu 450 Milliarden US-Dollar möglich [3]. Dies gilt analog für Deutschland und Europa. Diese wirtschaftliche Bedeutung steht im Gegensatz zur Investitionsbereitschaft in die Informationstechnik (IT) und zum allgegenwärtigen Kostendruck. Obwohl technische Lösungen zur Integration und Verwertung der Daten bereitstehen, ist auch Deutschland ein Land der Insellösungen und Silo-Politik, das heißt insbesondere, dass die Daten von Forschung und Entwicklung in den Lebenswissenschaften nicht durchlässig und transparent mit den Daten der Versicherungen, der Krankenhäuser, Ärzte und Patienten im Gesundheitssystem verknüpft sind. Es wäre wichtig, die Daten im Gesundheitssystem für ergebnisbasierte Forschung und Entwicklung von Anfang an nutzen zu können und umgekehrt.

Erste positive Beispiele von Big DataAnwendungen sind klar dokumentiert, z. B. die in nur drei Jahren erfolgte Entwicklung und Zulassung des Medikaments Xalkori für Lungenkrebspatienten mit einer Mutation des ALK-Proteins [4].

Die Politik kann mit den gesellschaftlichen Entwicklungen durch Social Media und der Verknüpfung zu E-Health und Mobile Health kaum Schritt halten, da diese breiten gesellschaftlichen Trends (Quantified Self) die bisherigen regulatorischen und datenschutzrechtlichen Regelungen ad absurdum führen. Eine Überarbeitung der datenschutzrechtlichen Bestimmungen unter Beachtung der Prinzipien der informationellen Selbstbestimmung und der Vermeidung des gläsernen Patienten sollte daher in Angriff genommen werden.

Die Herausforderungen von Big Data wollen zeitnah und gemeinschaftlich bearbeitet und gelöst werden. Im Oktober 2014 wurde im Verband Bio Deutschland eine neue Arbeits- gruppe „Bio-IT und Big Data“ gegründet, um das Wissen und die Interessen in diesem Bereich von Unternehmern aus Biotech, Pharma, Vertretern der Bioregionen, Universitäten und führenden akademischen Instituten, des Technologietransfers, Firmen aus dem IT-Sektor im Rahmen von Branchenpartnerschaften und Juristen sowie Wirtschaftsprüfern zu bündeln. Die Arbeitsgruppe soll u. a. politische Handlungsempfehlungen im Rahmen eines Positionspapiers erarbeiten und neben dem Dialog mit der Politik und der Öffentlichkeit verstärkt den Kontakt zu anderen Akteuren im Gesundheitswesen wie Patienten, Ärzten, Kliniken und Krankenversicherungen suchen.

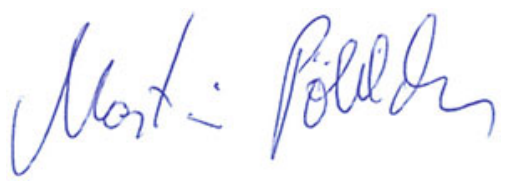

Dr. Martin Pöhlchen,

Geschäftsführer Sinfonie Life Science Management GmbH und Leiter AG Bio-IT und Big Data, Bio Deutschland

\section{Links}

[1] http://blogs.gartner.com/douglaney/files/2012/01/ad949-3D-Data-Management-ControllingData-Volume-Velocity-and-Variety.pdf

[2] http://de.slideshare.net/schappy/gaining-time-realttimeanalysis-of-big-medical-data

[3] www.mckinsey.com/insights/health_systems_and_services/the_big-data_revolution_in_us_health_care

[4] www.mckinsey.com/ $\sim$ media/mckinsey/dotcom/client service/Pharma\%20and\%20Medical\%20Products/PMP\%2 0NEW/PDFs/McKinsey\%20on\%20Personalized\%20Medicine\% 20March\%202013.ashx

\section{Korrespondenzadresse:}

Dr. Martin Pöhlchen

Sinfonie Life Science Management $\mathrm{GmbH}$

Semmelweisstraße 8

D-82152 Planegg

Tel.: 089-858963-162

Fax: 089-858963-163

mp.sinfonie@t-online.de 